\title{
ANÁLISIS
}

\section{La dimensión institucional en la relación ANSEA-OCS ${ }^{1}$}

DOI: $10.32870 /$ mycp.v2i3.396

Francisco Javier Haro Navejas ${ }^{2}$

Rosángel Hernández Mendoza ${ }^{3}$

\section{Resumen}

El trabajo analiza las relaciones -en sus niveles formal e informal- entre la Asociación de Naciones del Sudeste de Asia (ANSEA) y la Organización para la Cooperación de Shanghai (ocs), enfatizando los elementos que vinculan a ambas organizaciones en una relación de aprendizaje e imitación. Con base en este análisis, los autores discuten los procesos de institucionalización y socialización que ocurren entre ambas organizaciones, así como el papel de China como vínculo entre Asia central y el sudeste de Asia. Finalmente, exponen algunas ideas para entender la relación entre la ANSEA y la OcS en el contexto del proceso de desarrollo de las relaciones político-diplomáticas en el sudeste y centro de Asia. En el trabajo, de fuerte orientación institucionalista, se recuperan planteamientos constructivistas, sobre todo en lo que se refiere a los tipos de identidad colectiva.

Palabras clave: Organización de Cooperación de Shanghai, Asociación de Naciones del Sudeste de Asia, China, socialización, identidad colectiva.

1. Artículo recibido el 30 de octubre de 2012 y dictaminado el 15 de diciembre de 2012.

2. Es coordinador de Diez naciones en busca de liderazgo. Entre sus trabajos más recientes están The People's Republic of China in Central America and the Caribbean: Reshaping the region; Beijing frente a las 'minorías nacionales': la fe grande y las fes pequeñas; La identidad como eje del conflicto Beijing-Taipei. Actualmente es profesor investigador de la UCOL. ORCID http://orcid.org/0000-0002-1061-2508

3. Egresada de la Maestría en Estudios de Asia y África, con especialidad en el área de China, del Centro de Estudios de Asia y África de El Colegio de México. Ha realizado estudios de idioma en la República Popular China. 


\section{Abstract}

This paper analyzes the formal and informal relations between the Association of Southeast Asian Nations (ASEAN) and the Shanghai Cooperation Organization (sco). It emphasizes elements which link the two organizations in a relationship of learning and imitation. Based on this analysis, the authors discuss the institutionalization and socialization processes that occur between the two organizations, as well as China's role as a link between Central and Southeast Asia. Finally, they introduce some ideas for understanding the relationship between ASEAN and SCO in the context of the development of political and diplomatic relations in Southeast and Central Asia. The paper has a strong institutionalist orientation and it employs constructivist approaches, especially regarding the types of collective identity.

Keywords: Shanghai Cooperation Organization, Association of Southeast Asian Nations, China, socialization, collective identity.

\section{Introducción}

En gran parte de Asia, sobre todo en el sur, centro y este, hay un boom cualitativo institucional sin precedentes. El entusiasmo es tal que, en el este, se habla ya del surgimiento de una "identidad asiática" (Tay, 2009). Las organizaciones regionales más destacadas son: la Asociación de Naciones del Sudeste de Asia (ANSEA), fundada en 1967; la Asociación para la Cooperación Regional del Sur de Asia (ACRSA), creada en 1985, y la Organización para la Cooperación de Shanghai (OCS), que tomó forma en 2001. De acuerdo a algunos especialistas, como Shambaugh (2005: 11), el Foro Regional de la ANSEA ${ }^{4}$ es la base de esta "comunidad regional emergente", y las otras dos son "partes componentes importantes".

4. El Foro Regional de la ANSEA (ARF, por sus siglas en inglés) fue concebido por los Estados miembros de la ANSEA como un medio para impulsar el diálogo y la consulta sobre asuntos políticos y de seguridad, a fin de desarrollar medidas generadoras de confianza y mecanismos de diplomacia preventiva para la región. Su primera reunión se celebró en 1994 con la participación de los representantes de 18 países: los entonces seis estados miembros de la ANSEA (Brunei, Indonesia, Malasia, Filipinas, Singapur y Tailandia), sus siete socios de diálogo (Australia, Canadá, la Unión Europea, Japón, Nueva Zelanda, Corea del Sur y Estados Unidos), sus dos socios consultivos (China y Rusia) y cuatro Estados observadores (Laos, Papua Nueva Guinea y Vietnam). 
Dada la creciente importancia que ha cobrado la dimensión institucional en la región y la ausencia de estudios al respecto, estas páginas se enfocan a analizarlo en el contexto de las relaciones entre la ANSEA y la Ocs. El objetivo de este trabajo es analizar cómo interactúan institucionalmente el sudeste de Asia y Asia central. Para llevar a cabo dicho análisis, distinguimos dos tipos de relaciones: la relación formal, establecida en 2005 con la firma del Memorando de Entendimiento entre el Secretariado de la ANSEA y el Secretariado de la OCS, y la relación informal. Ambas permiten apreciar principalmente las semejanzas entre las dos organizaciones.

En este análisis consideramos que el proceso de formación de dichas organizaciones sucede en un contexto de conformación de un orden regional donde los Estados crean nuevas reglas del juego alrededor de instituciones ad hoc, ya sea que éstas sean reformadas o nuevas. Para ubicar el análisis en este contexto, la perspectiva conceptual del presente trabajo, retomada de Wendt (1994) y de Haro (2005), ${ }^{5}$ considera que la ausencia y/o presencia de instituciones intergubernamentales debe ser analizada dentro de contextos históricos específicos, sobre todo de acuerdo a las formas que adquieren las relaciones entre los Estados y sus identidades, las cuales no surgen de una vez y para siempre; por el contrario, se transforman. ${ }^{6}$

Esto se debe a que el proceso de formación de identidades e instituciones ocurre en ambos sentidos: los países construyen organizaciones internacionales a las cuales modifican de acuerdo a sus cambios identitarios; al mismo tiempo, sus propias conductas e identidades son modificadas por ellas, es decir, son socializados. ${ }^{7}$ Factores internos y externos se combinan para conducir a los Estados a reformar, caso de la ANSEA, o edificar, situación de la Ocs, instituciones que sirvan, en primer lugar, para forjar la cooperación que lleve a obtener mayores beneficios con costos más reducidos; segundo, a incrementar

5. Wendt (1994) plantea que la formación de una identidad colectiva es imprescindible para la acción colectiva y el surgimiento de Estados internacionales. Por su parte, Haro (2005) propone utilizar un "constructivismo institucionalista" para analizar el proceso de definición de identidades que llevó al gobierno chino a impulsar el establecimiento de la ocs. La unión de ambos planteamientos permite formar una perspectiva conceptual que enfatiza la importancia de las identidades en la construcción de instituciones.

6. No discutimos las diferentes perspectivas teóricas para el estudio de Asia. Una excelente muestra del debate está en Acharya (1999) y en Narine (2000).

7. En el ámbito de las relaciones internacionales, se conoce como "socialización" al proceso mediante el cual el gobierno de un Estado interioriza las creencias, normas, actitudes y comportamientos aceptados y practicados en el sistema internacional o por los miembros de un grupo determinado. El tema ha sido abordado por Johnston (2003) y por Haro (2006). 
Factores internos y externos se combinan para conducir a los Estados a reformar, caso de la ANSEA, o edificar, situación de la ocs, instituciones que sirvan, en primer lugar, para forjar la cooperación que lleve a obtener mayores beneficios con costos más reducidos; segundo, a incrementar la predictibilidad; $y$, en última instancia, a la formación de una identidad colectiva

la predictibilidad; y, en última instancia, a la formación de una identidad colectiva (Keohane, 1984).

En la relación institucional analizada en estas páginas, se podrá apreciar que, tanto al interior de cada una de las organizaciones como entre ellas, se avanza en los dos primeros aspectos, menos costos y mayor predictibilidad; sin embargo, la identidad es hasta ahora fuerte solamente a nivel discursivo. Para conducir este análisis, el presente trabajo

se divide en cinco secciones. En la primera, presentamos la relación informal entre la ANSEA y la OCS, es decir, los elementos que vinculan a ambas organizaciones en una relación de aprendizaje e imitación. En la segunda, discutimos el estado actual de sus relaciones formales en los ámbitos político-diplomático y comercial. En la tercera, analizamos el estadio de desarrollo de la identidad colectiva de la ANSEA y la OCS. En seguida, en la cuarta, nos concentramos en los procesos de institucionalización y socialización por los que han atravesado dichas organizaciones, así como el papel de China como vínculo entre ambas regiones. Por último, en la quinta sección concluimos este análisis ofreciendo algunas ideas para entender la relación entre la ANSEA y la ocs en el contexto del proceso de desarrollo de las relaciones político-diplomáticas en el sudeste y centro de Asia.

\section{La relación informal: aprendizaje e imitación}

\subsection{ANSEA, imán y modelo institucionalista}

La ANSEA, fundada el 8 de agosto de 1967, inició básicamente alrededor de dos ejes, estrechamente entrelazados: uno, ideológico, el anticomunismo; y otro, práctico, convertirse en uno de los elementos de un amplio esquema de 
seguridad alimentado por Estados Unidos. La heterogeneidad, desde cualquier variable, como parcialmente se aprecia en el cuadro 1, ha sido el sello de la institución. Lo cual se ha acentuado con los miembros más nuevos, en contra de quienes, salvo Brunei, estaba enderezado el bloque que evitaría la caída del dominó a fines de los años sesenta: Camboya, Laos y, sobre todo, Vietnam.

\section{Cuadro 1}

Datos básicos de los países miembros de ANSEA

\begin{tabular}{llrr}
\hline País & Ingreso & \multicolumn{1}{c}{ Área } & Población (miles) \\
\hline Brunei & 8 de enero, 1984 & $5,765 \mathrm{~km}^{2}$ & 370.1 \\
Camboya & 30 de abril, 1999 & $181,035 \mathrm{~km}^{2}$ & $13,661.4$ \\
Indonesia & 8 de agosto, 1967 & $1,890,754 \mathrm{~km}^{2}$ & $219,205.0$ \\
Filipinas & 8 de agosto, 1967 & $300,000 \mathrm{~km}^{2}$ & $85,236.9$ \\
Laos & 23 de julio, 1997 & $236,800 \mathrm{~km}^{2}$ & $5,938.8$ \\
Malasia & 8 de agosto, 1967 & $330,257 \mathrm{~km}^{2}$ & $26,127.7$ \\
Myanmar & 23 de julio, 1997 & $676,577 \mathrm{~km}^{2}$ & $56,002.6$ \\
Singapur & 8 de agosto, 1967 & $697 \mathrm{~km}^{2}$ & 4.198 \\
Tailandia & 8 de agosto, 1967 & $513,254 \mathrm{~km}^{2}$ & $64,763.0$ \\
Vietnam & 23 de julio, 1997 & $330,363 \mathrm{~km}^{2}$ & $83,119.9$ \\
\hline
\end{tabular}

Fuente: elaborado con información procedente de: http://www.asean.org/.

Además de haber dejado atrás los atavismos ideológicos y de ampliar su membresía al aceptar a otros de sus vecinos, ${ }^{8}$ la ANSEA se ha convertido en el eje del institucionalismo regional, incluso desplazando considerablemente al Foro de Cooperación Económica Asia-Pacífico (APEC, por sus siglas en inglés), imán para otros países, especialmente fuera de la región asiática. Es el marco de una intensa competencia cooperativa en ANSEA + 3 (Corea del Sur, China y Japón), ANSEA + Cumbre con India, el Encuentro Asia-Europa, Foro Boao por Asia, Diálogo para la Cooperación de Asia; además, están sus lazos con APEC y, claro, con la Ocs. De acuerdo a ANSEA, mantiene diálogo con 11 socios principales: Australia, Canadá, Corea del Sur, China, Estados Unidos, Federación Rusa, India, Japón, Naciones Unidas (para programas relacionados con

8. Después de su establecimiento en 1967, Brunei ingresó a la ANSEA en 1984, Vietnam en 1995, Laos y Myanmar en 1997 y Camboya en 1999. 
el desarrollo), Nueva Zelanda, y la Unión Europea (UE). Asimismo, hace algo similar — pero aún no tan formal— con Pakistán. Finalmente, ha establecido estrechas relaciones con sus pares de otras zonas del planeta, como el Consejo para la Cooperación del Golfo, el Foro del Pacífico Sur, el Grupo de Río y la Conferencia para la Organización Subregional Afro-asiática.

El aspecto más importante de ANSEA, como punto de referencia socializador institucional, es la capacidad para servir como marco donde no solamente los intereses específicos, egoístas, se expresan y defienden, sino que permite la definición de un "interés general colectivo" (Ramírez Bonilla, 2012). Por lo tanto, su importancia para el escenario multilateral de la región radica en dos aspectos esenciales: sus políticas exitosas de integración, sustentadas en la mencionada desideologización, y ser el elemento primordial para la reorganización de la relación de fuerzas entre los poderes establecidos, Estados Unidos y Japón, y los de reciente aparición, China.

El otro aspecto relevante es el organizativo, que se distingue principalmente por su relativa simplicidad, lo cual le imprime flexibilidad y le quita peso burocrático, haciendo recaer la mayor parte de sus tareas sobre las burocracias nacionales coordinadas por su Secretariado. En el organigrama 1 se aprecian las características mencionadas, donde formalmente la autoridad se otorga a la reunión anual de los jefes de Estado y/o de gobierno. En los hechos, el trabajo es realizado por los subcomités y grupos de trabajo de los diversos ministerios, especialmente los de economía y de asuntos exteriores. ${ }^{9}$

La desideoligización de la ANSEA ha tenido como una de sus facetas principales la ampliación de su agenda, en la cual sigue ocupando un lugar central la seguridad; sin embargo, está lejos de ser el único elemento o el más importante. Incluso, en materia de seguridad se ha avanzado para ir de una alianza mantenida a fin de cuentas por los norteamericanos, al impulso al desarrollo de una comunidad regional, ligada ahora a temas como democracia y protección del medio ambiente. En relación a lo anterior, debemos considerar que no solamente pretenden construir una comunidad de seguridad, sino también crear comunidades en dos campos:

1. El económico, donde se busca fortalecer la integración comercial y a los mecanismos institucionales, sobre todo en materia de solución pacífica de controversias.

9. Todo lo relativo a estructura y funcionamiento de ANSEA se encuentra en: http://www.asean.org/. 


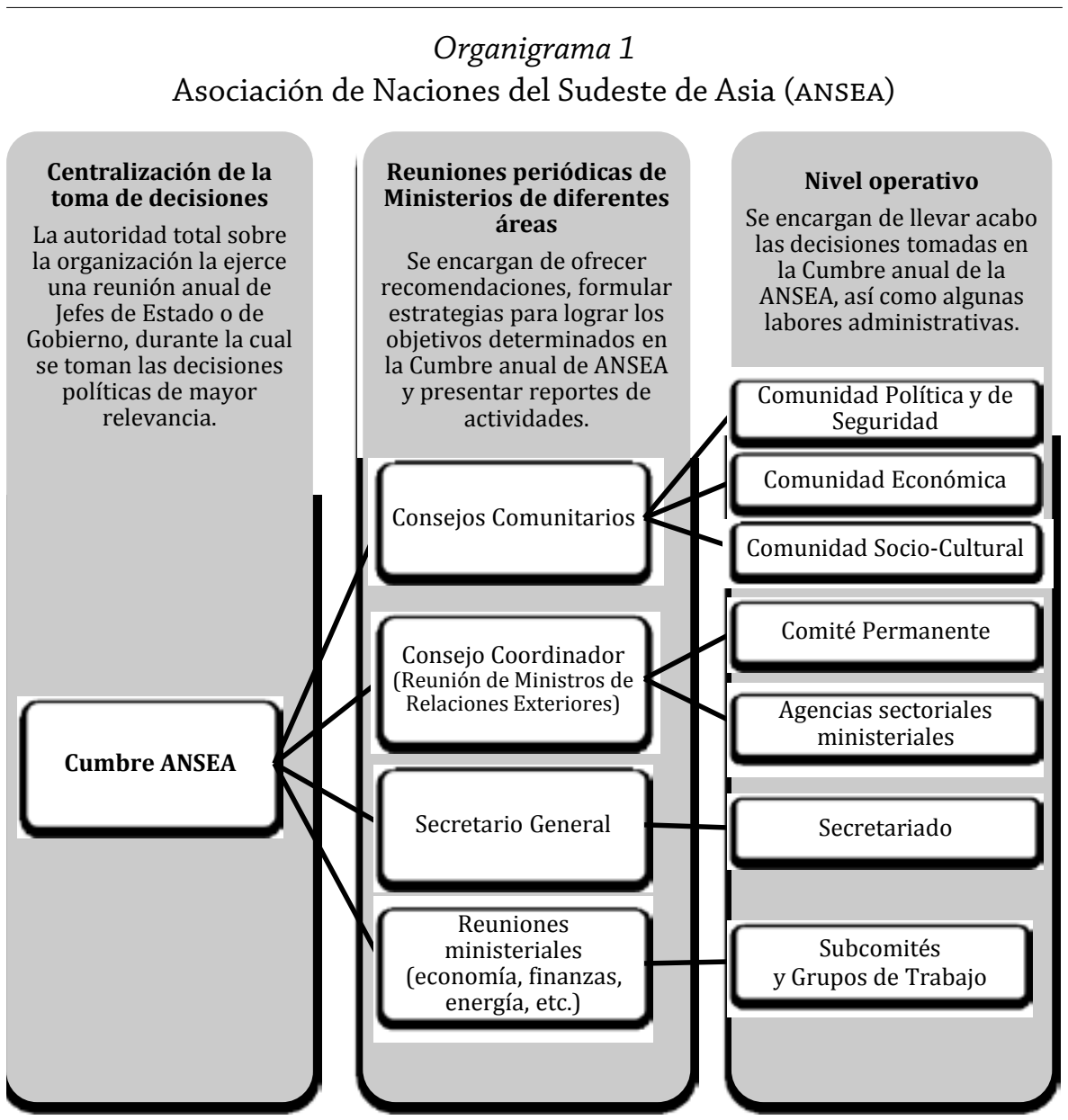

Fuente: elaborado con información de: http://www.asean.org/asean/asean-structure.

2. El social, para el que pretenden construir una identidad regional, reforzando ciertos aspectos socio-culturales comunes e impulsando el desarrollo — social y económico - regional. En este rubro, trabajan en la cooperación en materia de salud, en el desarrollo social para elevar los niveles de vida, y en la mayor inversión para educar mejor a su fuerza de trabajo. ${ }^{10}$

10. En 2003, durante la novena Cumbre de ANSEA, sus líderes decidieron formar una Comunidad ANSEA compuesta por tres pilares: la comunidad política y de seguridad, la comunidad económica 
La idea de una identidad común parece algo lejano, sobre todo debido a lo que se pudiera entender por ello, pero también por lo extenso de la región. Las enormes diferencias, la ampliación de la agenda, la mayor institucionalización y la creciente competencia cooperativa, conducen a ANSEA por el camino de la integración cada vez más profunda, pero sobre todo más institucionalizada.

\section{2 ocs, nacimiento del institucionalismo regional en Asia central}

Al igual que el sudeste de Asia, la parte central es muy heterogénea no importa la variable tomada como referencia comparativa, como se aprecia en el cuadro 2.

\section{Cuadro 2}

Datos básicos de los miembros de la ocs

\begin{tabular}{llll}
\hline País & Ingreso & Área & Población(millones) \\
\hline China & 14 de junio, 2001 & $9,600,000 \mathrm{~km} 2$ & 1,300 \\
Kazajistán & 14 de junio, 2001 & $2,717,300 \mathrm{~km} 2$ & 15.1 \\
Kirguistán & 14 de junio, 2001 & $198,500 \mathrm{~km} 2$ & 5.1 \\
Rusia & 14 de junio, 2001 & $17,075,200 \mathrm{~km} 2$ & 143.4 \\
Tayikistán & 14 de junio, 2001 & $143,100 \mathrm{~km} 2$ & 7.1 \\
Uzbekistán & 14 de junio, 2001 & $447,700 \mathrm{~km} 2$ & 26.8 \\
\hline
\end{tabular}

Fuente: elaborado con información procedente de: www.sectsco.org.

Su historia de desencuentros no es menor a la de los países de ANSEA, sobre todo por el hecho de que por cientos de años, bajo diferentes contextos históricos, los pueblos de Asia central se han enfrentado tanto a Moscú como a Beijing, a veces de manera simultánea, en una lucha alrededor de identidades y símbolos, donde hasta ahora sobresale el islam como identidad hegemónica; y de elementos materiales, los energéticos. Los roces, por momentos violentos, empiezan a ser desplazados por la cooperación.

El camino de la ocs ha sido largo y por momentos muy complicado. Su antecedente inmediato es el grupo llamado "los Cinco de Shanghai", establecido en 1996 y del cual no formaba parte Uzbekistán. Fue un arduo trabajo, tanto en

y la comunidad socio-cultural. Para mayor información sobre el proyecto de Comunidad ANSEA, véase: http://www.asean.org/. 
lo bilateral como en lo multilateral, facilitado, tal vez paradójicamente, por la relativa debilidad de Rusia y la también relativa fortaleza de China; pero sobre todo, de acuerdo a la concepción teórica aquí adoptada, al elemento discursivo estructurado alrededor de lo cooperativo institucional, en este caso enfocado a dos necesidades, ambas construidas y promovidas por el gobierno chino: 1 . Cooperar en el mantenimiento de la seguridad regional, especialmente en lo que se refiere al combate contra el terrorismo, el separatismo y extremismo y 2. Institucionalizar dicha cooperación por medio de una organización regional. Ambas ideas provienen de una previa definición de identidades que llevaron al gobierno chino a iniciar un intenso activismo diplomático para promover el establecimiento de la ocs en Asia central (Haro, 2005: 194-196).

Kangas (1995: 283) sintetiza muy bien el contexto que permitió la institucionalización de la cooperación en dicha región: "[L]a necesidad de alcanzar estabilidad doméstica se ha trasladado al impulso de cooperación y seguridad regionales". El proceso institucionalizador ha sido rápido y menos complicado que en el sudeste, lo que se ha debido a los cambios contextuales de las relaciones entre los involucrados, a las modificaciones identitarias provocadas por la implosión soviética, al ascenso chino enfocado en lo económico y al ejemplo que ofrecieron los procesos de construcción institucional de APEC y ANSEA.

No se debe obviar que, de manera no siempre clara, Rusia y China no han renunciado a lograr una cierta bi-hegemonía tanto material como cultural en la región. En el contexto histórico actual, la disputa por la preeminencia se da dentro de marcos institucionales claramente definidos y funcionales, donde las ganancias son mayores que los costos para todos los participantes. Se trata de intercambios marcados por la reciprocidad y la equivalencia, lo que quiere decir que las ganancias, sobre todo entre disímiles, nunca podrán ser iguales pero tampoco son completamente insatisfactorias para los más débiles. ${ }^{11}$ En la mejor de las circunstancias, los intercambios se acercan más a la satisfacción de los intereses, los cuales se ven mejor cristalizados de forma institucionalizada.

La agenda, al igual que en ANSEA, es cada vez más multifacética y dirigida hacia la creciente integración regional, basada en acuerdos parciales en diferentes campos, lo mismo del comercio y las finanzas, que del turismo y el transporte; sin dejar de lado, en ningún momento, lo relativo a la energía o temas relacionados con la educación y la protección ambiental. La ampliación

11. Sobre el tema de la reciprocidad en relaciones internacionales, véase Keohane (1986). 
de tópicos es facilitada sobre todo por dos pilares: creciente confianza mutua $\mathrm{y}$ fortalecimiento institucional.

Al comparar los organigramas de ANSEA y de la ocs, se pueden constatar las similitudes. Aunque presentados de manera diferente, la idea del funcionamiento organizativo es fundamentalmente la misma: centralización de las decisiones políticas de gran relevancia en la reunión anual de líderes y organismos responsables de llevar acabo dichas decisiones, apoyados y/o supervisados por reuniones periódicas de ministros de diferentes áreas.

La OCs es igualmente poco burocratizada, pero se aprecia una tendencia a una mayor centralización y vigilancia de los organismos dirigentes, sobre todo en lo que a antiterrorismo se refiere. Menos ambiciosa que la ANSEA en materia identitaria, la institución de Asia central, bajo la conducción de un secretariado y de las burocracias nacionales, siempre bajo la dirección de los jefes de Estado y sus respectivos ministerios, tiene una mayor participación explícita de diferentes organismos y agencias estatales, que formalmente serían poderes independientes internos, como sería el caso de los parlamentos y de los poderes judiciales.

\section{La relación formal: reconocimiento mutuo y comercio}

Desde la doble perspectiva diplomática y legal-institucional, el punto más alto de la relación entre ambas regiones fue alcanzado en 2005, con la firma del Memorando de Entendimiento entre el Secretariado de la ANSEA y el Secretariado de la OCS, ${ }^{12}$ el cual fue signado el 21 de abril de 2005 por los secretarios de ambos organismos, On Keng Yong, de la primera, y Shang Deguang, de la segunda, diplomáticos oriundos de Singapur y China, respectivamente.

El documento, legalmente no vinculante en materia de derecho internacional, es relevante al menos por las siguientes razones fundamentales:

a) Representa el reconocimiento mutuo, lo cual es más importante para la OCS, ${ }^{13}$ y significa la disponibilidad de ambas instancias intergubernamentales para fortalecer el camino institucional interregional en Asia.

12. El contenido completo del memorando puede consultarse en: http://www.asean.org/images/ ASEAN-SCO-MOU.pdf.

13. Lo anterior debido a que, desde la perspectiva de algunos actores internacionales occidentales, como Estados Unidos, la ocs ha sido percibida como una "amenaza", mientras que la ANSEA ha sido considerada como una influencia positiva en el sureste de Asia. Por lo tanto, el reconocimiento 


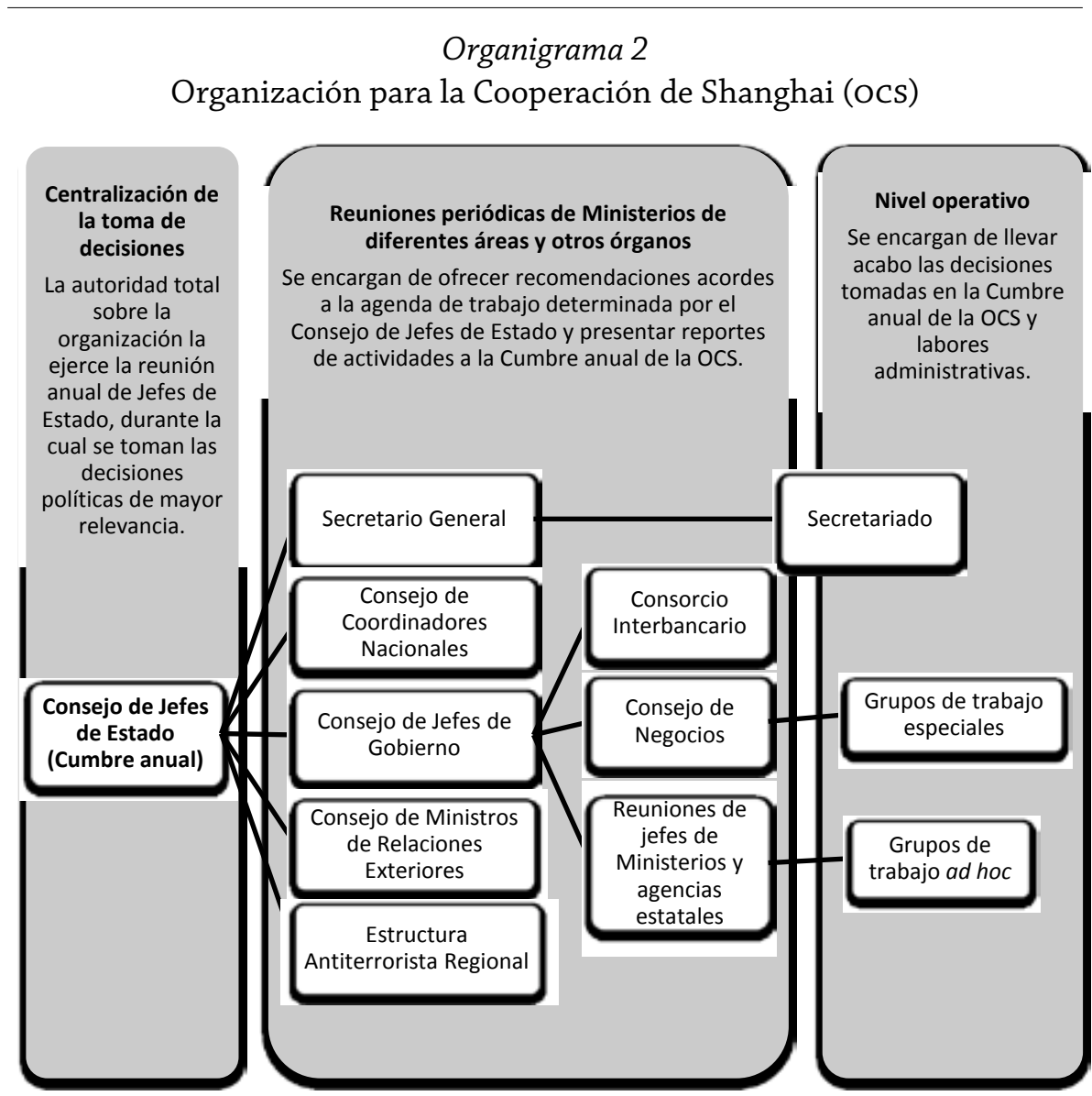

Fuente: elaborado con base en información de: http://www.sectsco.org/EN/.

b) Es la clausura implícita de una época de países enfrentados por razones ideológicas y prácticamente aislados entres sí durante décadas.

El memorando encapsula las preocupaciones de los gobiernos de la región, lo que quiere decir que se proponen cooperar en el combate al "crimen transnacional”: terrorismo, narcotráfico, contrabando de armas, lavado de dinero y

internacional que intercambiaron y el eventual inicio de una cooperación entre ambas servirá particularmente a la ocs para realzar su legitimidad internacional frente a ciertos actores occidentales (Aris, 2009: 464). 
tráfico de personas. Las experiencias de cooperación e integración, sobre todo por la travesía de la Comunidad Económica del Carbón y el Acero (CECA) a la UE, muestran que la única manera de avanzar es a partir de puntos concretos en los que sea relativamente sencillo ponerse de acuerdo, por lo menos en las primeras etapas integradoras. Al mismo tiempo, el texto abre la posibilidad para profundizar los esfuerzos de cooperación en economía y finanzas, turismo, medio ambiente y manejo de recursos naturales, desarrollo social, y energía.

Los mecanismos de cooperación son sencillos y baratos: intercambio de información, encuentros anuales, a veces informales, durante reuniones internacionales importantes, e intercambio de correspondencia cuando se trate de aspectos técnicos o sectoriales específicos. En lugar de seguir las caras y complejas tradiciones organizativas de la segunda posguerra, han optado por mecanismos simples y confiables, donde los costos se reducen al mínimo y las ganancias se podrían ampliar sin necesidad de crear más aparatos burocráticos que los que ya existen, tanto a nivel internacional como nacional. La negociación y firma de este memorando podría considerarse como un producto de la socialización exitosa de diferentes actores estatales alrededor de la ANSEA, la cual se ha convertido en un mecanismo para lograr la cooperación en un sinnúmero de aspectos y que sirve como modelo para procesos similares en Asia. Los actores no solamente aprenden e interiorizan que la cooperación es factible y útil en determinado espacio, sino que expanden la experiencia, y por razones lo mismo prácticas (costos), que políticas (lograr una mayor influencia), buscan evitar el aislamiento y/o la fragmentación de la región. Los fines están asociados lo mismo al beneficio colectivo que al individual, lo que significaría lograr mayor poder en la medida que se logra un cambio sustancial en el statu quo asiático. Lo anterior conduce, entre otros aspectos, al nacimiento de una China más poderosa, lo mismo en el centro que en el este. ${ }^{14}$

Pero no solamente Beijing busca un papel central en el desarrollo de los acontecimientos institucionales regionales, otros hacen lo propio. Filipinas ha saltado a la palestra para presentarse como el gobierno que lleve a una mayor profundidad la relación con Asia central. De hecho, desde septiembre de 2003, casi dos años antes de la firma del memorando, la entonces presidenta filipina Gloria Macapagal Arroyo, ya había expresado su interés por explorar formas de cooperación antiterrorista entre ANSEA y la OCS, en un comunicado conjunto realizado en ocasión de la visita a Manila de Wu Bangguo, entonces

14. El tema ha sido analizado parcialmente en Haro (2007). 
presidente del Comité Permanente de la Asamblea Popular Nacional de China. ${ }^{15}$ Posteriormente, en la $11^{\text {a }}$ Cumbre de ANSEA, celebrada en diciembre de 2005, Macapagal propuso directamente desarrollar una mayor cooperación entre la ANSEA y la OCS para la lucha contra el terrorismo (People's Daily Online, 2005). Un año después, aprovechando su condición de anfitriona de la $12^{\text {a }}$ Cumbre de la ANSEA (enero de 2007), Macapagal y la diplomacia filipina lograron incluir en la declaración final el compromiso de "forjar una cooperación más cercana" con la ocs, para lo cual el documento citado encomendó a sus funcionarios la tarea de "identificar los medios para fortalecer" los lazos con la OCS (ASEAN, 2007). Sin embargo, a la fecha, sigue por verse si la idea filipina se concretará en acciones específicas y si la visión del presidente Benigno Aquino III sobre este tema coincide con la de su antecesora, lo cual se complica con los cargos de corrupción que pesan sobre la anterior mandataria. Además, con el resurgimiento de las tensiones entre China y Filipinas sobre el arrecife Scarborough ${ }^{16}$ en abril de 2012, parece difícil prever que éste último retome el liderazgo para impulsar la cooperación ANSEA-OCS.

\subsection{La débil dimensión comercial}

Desde los años ochenta se ha puesto en el centro del debate económico al comercio como condición sine qua non de los procesos de integración y cooperación, lo que no necesariamente tiene que ser de esa manera dado lo cambiante de las agendas nacionales e internacionales. Sin duda, es un elemento importante, sobre todo en la regionalización. Sin embargo, en la medida que los países tienen límites en sus niveles de producción que les impide comerciar con todo el mundo en las mismas cantidades, los elementos discursivos alrededor de tópicos comerciales se convierten en el trampolín para la integración y la cooperación suprarregional. Por ejemplo, el proyecto de "reconstrucción de la Ruta de la Seda" que sirvió de tarjeta de presentación del gobierno chino durante la primera gira que un alto funcionario realizó en Asia central en 1994 (Beijing Review, 1994). Como en este caso, aunque en la práctica sea mínimo e incluso en teoría sea difícil incrementar, el incentivo del comercio seguirá siendo esencial ya que abre las posibilidades de poder

15. Dicho comunicado puede consultarse en: http://www.china.org.cn/english/international/73968. htm. Fecha de consulta: octubre 18, 2012).

16. Para China, Isla Huangyan. 
acceder a nuevos mercados, lo cual es una de las facetas más positivas de la economía internacional actual: la existencia de consumidores por conquistar. De hecho, si en algo difiere el contexto internacional actual de los existentes — por ejemplo, entre 1871 y 1914, o entre 1929 y 1939_, es precisamente en la debilidad actual del proteccionismo.

Si al estudiar las relaciones entre las naciones del sudeste asiático, principalmente las pertenecientes a ANSEA, y las de Asia central el análisis se circunscribiera al aspecto comercial el resultado de la investigación, nos llevaría a concluir que la relación es casi nula, como nos muestra el cuadro 3.

\section{Cuadro 3}

Socios comerciales de ANSEA en Asia central, 2010

\begin{tabular}{|c|c|c|c|c|c|c|c|}
\hline & \multicolumn{3}{|c|}{$\begin{array}{c}\text { Valor del comercio } \\
\text { Miles de dólares de Estados Unidos }\end{array}$} & \multicolumn{3}{|c|}{ Valor del comercio \% } \\
\hline & & Exportaciones & Importaciones & Comercio total & EXP & $I M P$ & $\begin{array}{l}\text { Comercio } \\
\text { total }\end{array}$ \\
\hline Miembros & China & $112,999,803.2$ & $119,013,395.5$ & $232,013,198.7$ & 10.6 & 12.2 & 11.3 \\
\hline \multirow[t]{3}{*}{ OCS } & Rusia & $2,589,972.1$ & $6,473,574.4$ & $9,063,546.5$ & 0.2 & 0.7 & 0.4 \\
\hline & Kazajistán & $150,512.5$ & $24,814.3$ & $175,326.8$ & 0.0 & 0.0 & 0.0 \\
\hline & Uzbekistán & $61,499.4$ & $22,915.1$ & $84,414.5$ & 0.0 & 0.0 & 0.0 \\
\hline \multirow{5}{*}{ 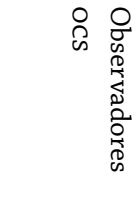 } & Afganistán & $244,018.4$ & $114,016.8$ & $358,035.3$ & 0.0 & 0.0 & 0.0 \\
\hline & India & $36,028,694.3$ & $19,414,714.5$ & $55,443,408.8$ & 3.4 & 2.0 & 2.7 \\
\hline & Irán & $2,064,797.2$ & $1,509,111.6$ & $3,573,908.9$ & 0.2 & 0.2 & 0.2 \\
\hline & Mongolia & $32,733.3$ & $7,140.0$ & $39,873.4$ & 0.0 & 0.0 & 0.0 \\
\hline & Pakistán & $5,113,843.6$ & $1,142,446.6$ & $6,256,290.2$ & 0.5 & 0.1 & 0.3 \\
\hline \multirow{3}{*}{$\begin{array}{l}\text { Socios de } \\
\text { diálogo } \\
\text { ocs }\end{array}$} & Sri Lanka & $1,184,752.5$ & $463,142.9$ & $1,647,895.4$ & 0.1 & 0.0 & 0.1 \\
\hline & Belarús & $17,307.4$ & $55,725.4$ & $73,032.8$ & 0.0 & 0.0 & 0.0 \\
\hline & Turquía & $2,807,326.7$ & $2,347,089.9$ & $5,154,416.6$ & 0.3 & 0.2 & 0.3 \\
\hline \multirow{2}{*}{$\begin{array}{l}\text { Otros } \\
\text { socios de } \\
\text { la región }\end{array}$} & Turkmenistán & $33,542.1$ & $2,697.5$ & $36,239.6$ & 0.0 & 0.0 & 0.0 \\
\hline & Azerbaiyán & $14,322.6$ & $55,019.2$ & $69,341.8$ & 0.0 & 0.0 & 0.0 \\
\hline
\end{tabular}

Fuente: información hasta el 15 de febrero de 2012, tomada de ASEAN Trade Database, disponible en: http://www.aseansec.org/stat/Table24_27.pdf. Fecha de consulta: julio 16, 2012).

Con excepción del caso de su relación comercial con China, los intercambios con el resto de sus socios en Asia central, incluyendo Rusia, son tan insignificantes que se representan con cero, en términos porcentuales. Lo cual 
es explicable en función de diferentes elementos conectados entre ellos, entre los que destacan los límites objetivos que los países enfrentan en sus niveles de producción-consumo. Aun cuando las naciones del sudeste de Asia desearan comerciar más, no lo pueden hacer. Lo que producen ya tiene más o menos sus mercados establecidos, por lo cual sería imposible que enviarán sus mercancías a otras naciones, al menos por ahora. La posibilidad de buscar nuevos mercados para lo que ahora se produce no se puede plantear ni siquiera como hipótesis, sobre todo debido a que la producción en gran medida se debe a la inversión extranjera directa, la cual en el sudeste de Asia sirve para exportar con menores costos a su lugar de origen o a otros mercados que le son conocidos.

La estructura del comercio en el este de Asia es esencialmente regional, lo cual puede apreciarse en el cuadro 4. Sin contar los intercambios con Estados Unidos, en la porción de Asia que nos ocupa estamos ante un proceso de competencia integradora. Los principales socios de ANSEA son, como economías individuales, Japón y China, pero si tomamos a ésta como parte de un conjunto integrado que incluye a Taiwán y Hong Kong estamos ante el socio más importante.

\section{Cuadro 4}

Principales socios comerciales de ANSEA, 2010

\begin{tabular}{lrrrrrr}
\hline & \multicolumn{3}{c}{ Valor del comercio } & \multicolumn{3}{c}{ Valor del comercio \% } \\
& \multicolumn{3}{c}{ Millones de dólares de Estados Unidos } & & \multicolumn{1}{c}{ Total } \\
& Exportaciones & Importaciones & $\begin{array}{c}\text { Comercio } \\
\text { toxp. }\end{array}$ & Imp. & $\begin{array}{c}\text { Comercio } \\
\text { total }\end{array}$ \\
\hline ANSEA & $267,981.0$ & $251,823.8$ & $519,804.7$ & 25.0 & 25.8 & 25.4 \\
China & $112,999.8$ & $119,013.4$ & $232,013.2$ & 10.6 & 12.2 & 11.3 \\
Unión Europea (27) & $115,036.4$ & $93,548.4$ & $208,584.8$ & 10.7 & 9.6 & 10.2 \\
Japón & $102,890.8$ & $103,746.3$ & $206,637.1$ & 9.6 & 10.6 & 10.1 \\
Estados Unidos & $100,464.7$ & $86,220.0$ & $186,684.7$ & 9.4 & 8.8 & 9.1 \\
Corea & $44,980.1$ & $53,648.2$ & $98,628.3$ & 4.2 & 5.5 & 4.8 \\
India & $36,028.7$ & $19,414.7$ & $55,443.4$ & 3.4 & 2.0 & 2.7 \\
Australia & $35,250.8$ & $20,175.4$ & $55,426.3$ & 3.3 & 2.1 & 2.7 \\
Hong Kong & $33,039.4$ & $21,292.3$ & $54,331.7$ & 3.1 & 2.2 & 2.7 \\
Taiwán & $16,122.3$ & $18,989.3$ & $35,111.6$ & 1.5 & 1.9 & 1.7 \\
Otros & $206,147.4$ & $186,917.8$ & $393,065.2$ & 19.2 & 19.2 & 19.2 \\
Total & $1,070,941.4$ & $974,789.6$ & $2,045,731.0$ & 100.0 & 100.0 & 100.0 \\
\hline
\end{tabular}

Fuente: Datos al 15 de febrero de 2012, tomados de ASEAN Trade Database. Disponible en: http://www.aseansec.org/stat/Table20_27.pdf. Fecha de consulta: julio 16, 2012). 
Lo que la información disponible nos muestra es que los intereses comerciales de ANSEA están centrados en el noreste y sur de Asia, pero de ninguna manera en Asia central o con los miembros de la ocs. A las razones anteriores, y al hecho de que históricamente el este y en menor medida el sur han estado más integrados entre sí, se podría señalar que bajo las actuales circunstancias la región central no cuenta con los medios para convertirse en un socio comercial considerable de ANSEA o de casi ningún otro país, ya que por el momento está sujeta a las posibilidades que les abra principalmente China.

En cuanto a la estructura del comercio de los miembros de la ocs, cabe destacar que ésta también es preponderantemente regional, y de marcada dependencia hacia los mercados ruso y chino. Fenómeno que puede observarse en el cuadro 5 .

\section{Cuadro 5}

Principales socios comerciales de los miembros de la ocs, 2010

\begin{tabular}{lll}
\hline & Socio / Exportaciones (\%) & Socio / Importaciones (\%) \\
& Millones de dólares de Estados Unidos & Millones de dólares de Estados Unidos \\
\hline China & Estados Unidos / 283,678.83 (17.95) & Japón / 176,304.03 (12.65) \\
& Hong Kong / 218,204.97(13.81) & Corea / 138,023.82 (9.90) \\
& Japón / 120,262.43 (7.61) & Estados Unidos / 101,959.20 (7.31) \\
Kazajistán & China / 10,031.29 (21.08) & China / 10,207.09 (34.14) \\
& Alemania / 4,499.23 (9.46) & Rusia / 5,582.02 (18.67) \\
& Francia / 3,550.15 (7.46) & Alemania / 1934.67 (6.47\%) \\
Kirguistán & Rusia / 351.77 (31.94) & China / 4,509.45 (62.77) \\
& Uzbekistán / 257.89(23.42) & Rusia / 1,072.91 (14.94) \\
& Kazajistán / 204.52 (18.57) & Kazajistán / 418.44 (5.82) \\
Rusia & Países Bajos / 53,240.38 (14.25) & China / 39, 059.01 (17.96) \\
& Italia / 24, 375.90 (6.52) & Alemania / 26,620.34 (12.24) \\
& China / 19,783.24 (5.29) & Ucrania / 14,002.53 (6.44) \\
Tayikistán & China / 446.96 (37.41) & Rusia / 856.53 (32.26) \\
& Turquía / 376.96 (31.55) & Kazajistán / 292.70 (11.02) \\
& Rusia / 101.81 (8.52) & China / 238.17 (8.97) \\
Uzbekistán & Rusia / 1,375.89 (24.69) & Rusia / 1,829.92 (21.43) \\
& China / 1,181.10(21.19) & Corea / 1,582.51 (18.54) \\
& Turquía / 783.07 (14.05) & China / 1,295.90 (15.18) \\
\hline
\end{tabular}

Fuente: elaboración propia con base en datos de la Dirección de Estadísticas de Comercio (DOTS, por sus siglas en inglés) del Fondo Monetario Internacional. Disponible en: http://elibrary-data.imf.org/FindDataReports.aspx?d=33061\&e=170921. Fecha de consulta: julio 17, 2012). 


\section{El discurso de la identidad}

De acuerdo con la perspectiva conceptual adoptada en este trabajo, la interacción de los Estados al interior de una organización o sistema internacional puede llevar a la modificación de sus intereses e identidades. La formación de una identidad colectiva, es decir, una identidad compartida por diferentes Estados que participan en un determinado arreglo institucional, permitiría el surgimiento de "Estados internacionales"17 y, a la vez, ayudaría a librar los obstáculos principales para la acción colectiva eficaz en el ámbito internacional (Wendt, 1994: 384-386). Asumiendo que la acción colectiva y coordinada frente a ciertos temas y problemas es la meta principal de las organizaciones, la formación de su identidad colectiva se convierte en un requisito indispensable para operar de manera igualmente adecuada y eficaz frente a cualquier tipo de problema.

Para facilitar el análisis del proceso de formación de la identidad colectiva, Wendt (1994: 385-386) distingue entre dos tipos de identidad: la identidad corporativa, que se refiere a las características concretas y estructurales de un grupo, y la identidad social, la cual se define por medio de un conjunto de significados que le permiten a un actor, por un lado, definir su papel en un contexto social específico; por el otro, formar intereses e identidades colectivas. Esta distinción nos ayuda a identificar el estadio de la identidad colectiva de nuestras dos organizaciones y nos permite concluir que los principales elementos que conforman su identidad corporativa se encuentran en mayor o menor medida definidos. Como se observa en el cuadro 6, estos elementos se refieren a las características básicas que han sido aceptadas por todos a través de la firma de ciertos documentos, como cartas o declaraciones, que formalizan la forma de operación y los objetivos de su asociación.

A diferencia de la identidad corporativa, la identidad social es apenas una aspiración para ambas organizaciones. En el caso de ANSEA, el proyecto ha tomado la forma de iniciativas como la Comunidad ANSEA, pero se remonta a acuerdos como el Tratado de Amistad y Cooperación en el Sudeste de Asia, que señala los principios que deben guiar sus relaciones y conducta internacional. A pesar de estos y otros esfuerzos, los Estados miembro de ANSEA no han logrado consensuar su posición institucional frente a temas fundamentales

17. Estructuras transnacionales con una autoridad política descentralizada. Para mayores detalles, véase Wendt (1994: 392). 


\begin{tabular}{|c|c|c|c|}
\hline \multicolumn{4}{|c|}{$\begin{array}{c}\text { Cuadro } 6 \\
\text { Identidad corporativa }\end{array}$} \\
\hline & ANSEA & \multicolumn{2}{|l|}{ OCS } \\
\hline Fundación & $\begin{array}{l}\text { 1967, en Bangkok, por } \\
\text { medio de la firma de una } \\
\text { Declaración }\end{array}$ & \multicolumn{2}{|c|}{$\begin{array}{l}\text { 2001, en Shanghai, con la firma de } \\
\text { una Declaración }\end{array}$} \\
\hline $\begin{array}{l}\text { Miembros } \\
\text { fundadores }\end{array}$ & $\begin{array}{ll}\text { Indonesia } & \text { Singapur } \\
\text { Malasia } & \text { Tailandia } \\
\text { Filipinas } & \end{array}$ & $\begin{array}{l}\text { China } \\
\text { Kazajistán } \\
\text { Kirguistán }\end{array}$ & $\begin{array}{l}\text { Rusia } \\
\text { Tayikistán } \\
\text { Uzbekistán }\end{array}$ \\
\hline Máximo órgano & $\begin{array}{l}\text { Cumbre anual de jefes de } \\
\text { Estado o de Gobierno }\end{array}$ & \multicolumn{2}{|c|}{ Cumbre anual de jefes de Estado } \\
\hline $\begin{array}{l}\text { Sede de su } \\
\text { secretariado }\end{array}$ & Jakarta, Indonesia & \multicolumn{2}{|c|}{ Beijing, China } \\
\hline $\begin{array}{l}\text { Número de } \\
\text { miembros }\end{array}$ & 10 & \multicolumn{2}{|l|}{6} \\
\hline Observadores & $\begin{array}{l}\text { Mantiene relaciones, bajo } \\
\text { diferentes modalidades, con } \\
\text { Australia, Canadá, China, la } \\
\text { Unión Europea, India, Japón, } \\
\text { República de Corea, Nueva } \\
\text { Zelanda, Rusia y Estados } \\
\text { Unidos. }\end{array}$ & $\begin{array}{l}\text { Afganistán } \\
\text { India } \\
\text { Pakistán }\end{array}$ & $\begin{array}{l}\text { Irán } \\
\text { Mongolia }\end{array}$ \\
\hline Principal documento & $\begin{array}{l}\text { Carta, legalmente vinculante, } \\
\text { entró en vigor el } 15 \text { de } \\
\text { diciembre de } 2008\end{array}$ & \multicolumn{2}{|c|}{ Carta, adoptada en 2002} \\
\hline Objetivo declarado & $\begin{array}{l}\text { “Acelerar el crecimiento } \\
\text { económico, el progreso social } \\
\text { y el desarrollo cultural de la } \\
\text { región a través de esfuerzos } \\
\text { conjuntos en el espíritu de } \\
\text { igualdad y asociación, con } \\
\text { el fin de fortalecer las bases } \\
\text { de una comunidad próspera } \\
\text { y pacífica de Naciones del } \\
\text { Sudeste Asiático.” }\end{array}$ & \multicolumn{2}{|c|}{$\begin{array}{l}\text { "Fortalecer la confianza mutua, la } \\
\text { amistad y la buena vecindad entre } \\
\text { los Estados Miembros; fomentar la } \\
\text { cooperación en materia de energía } \\
\text { política, economía y comercio, } \\
\text { científica y tecnológica, cultural, } \\
\text { educativa, comunicaciones, medio } \\
\text { ambiente y otros campos; dedicarse } \\
\text { conjuntamente a conservar y } \\
\text { salvaguardar la paz regional, seguridad } \\
\text { y estabilidad." }\end{array}$} \\
\hline
\end{tabular}

Fuente: elaboración propia con información de http://www.aseansec.org/ (para el caso de la ANSEA) y http://www.sectsco.org/EN/ (para la Ocs). 
como las disputas territoriales en el mar del sur de China. Al respecto, basta recordar el fracaso de la reunión de la ANSEA sostenida en Camboya en julio de 2012, donde el anfitrión prefirió apoyar la postura china de continuar tratando el problema en el marco de "disputas bilaterales" (Perlez, 2012).

Desde su nacimiento, el proyecto de identidad regional de la ocs ha girado en torno al combate a los "tres males": terrorismo, separatismo y extremismo. Después de la declaración de establecimiento de la ocs, el segundo documento que firmaron fue la Declaración sobre el Combate al Terrorismo, el Separatismo y el Extremismo y el primer órgano que establecieron fue la Estructura Regional Antiterrorista. Al interior de la organización, este discurso lo han materializado por medio de ejercicios militares multilaterales en los que trabajan en una "respuesta conjunta" para diferentes amenazas a la seguridad regional. El primero de ellos fue "Coalición" en 2003 (el primer ejercicio militar multilateral en el que participó el ejército chino) y el más reciente, Misión de Paz 2012 (CCTV.COM English, 2012). Al exterior, aunque no lograron acordar una posición común frente a la lucha contra el terrorismo iniciada por Estados Unidos en 2001; en 2005, durante en su Cumbre Anual de jefes de Estado emitieron una declaración conjunta donde pidieron a la Coalición Antiterrorista en Afganistán que estableciera un calendario para su salida de las bases en territorio de los miembros de la ocs. De esta manera, la identidad social de la ocs parece apoyarse en elementos militares y territoriales directamente relacionados con la seguridad nacional e, indirectamente, con la de cada uno de los regímenes. No obstante, debido a que la influencia de la osc en el escenario internacional es aún limitada, la fortaleza de la identidad social de la ocs no ha sido verdaderamente probada aún.

\section{La relativa fortaleza del nivel institucional: socialización e institucionalización}

La relativa y diferenciada fortaleza del nivel institucional en ambas regiones, es producto de varios factores, algunos ya enunciados. Los más importantes son los cambios contextuales en los cuales se han dado las relaciones entre los actores estatales, con los consecuentes cambios identitarios y de las percepciones mutuas. El contexto regional ha favorecido la cooperación con fuertes elementos discursivos y una agenda no comercial producto de nuevas situaciones globales y regionales que se han conjuntado en un interés común 
por desarrollar condiciones de certidumbre en el escenario regional y preservar los regímenes, especialmente en el caso de la ocs. ${ }^{18}$

Como hemos anotado, la relación entre ambas regiones se da en dos niveles. Por un lado, el informal, donde se ha llevado a cabo un proceso de socialización indirecta: ANSEA se ha posicionado como un modelo de cooperación regional, cuya estructura y prácticas se han trasladado, por medio de China, hacia la ocs. Por otro lado, en el segundo nivel, el formal, ocurre el desarrollo del andamiaje legal que permitirá sentar las bases para una mayor cooperación entre esas dos regiones de Asia. En este nivel, los estadios de las relaciones, a grandes rasgos, se encuentran en acercamientos a nivel de observación, firma de un memorando y búsqueda de mecanismos para profundizar la relación.

A nivel institucional, la socialización se produce por medio de dos vías: directa e indirecta. La primera es la socialización de los miembros dentro de cada una de las organizaciones, de acuerdo a las reglas, códigos y símbolos que se crean para la interacción; mientras que la segunda ocurre entre las organizaciones, básicamente se da a través de cooperación, imitación y aprendizaje.

Para el tema que nos ocupa, la socialización indirecta, tenemos el ejemplo del resurgimiento de la ANSEA, su énfasis en el regionalismo abierto y su estructura organizativa simple que han servido de guía para la estructuración de la ocs. Sin duda, entre ambos procesos de desarrollo institucional hay vasos comunicantes muy importantes; como muestra de ello, basta considerar las similitudes entre el Tratado de Amistad y Cooperación en el Sudeste de Asia y el Tratado de Cooperación, Buena Vecindad y Amistad de Largo Plazo firmado en agosto de 2007 por los miembros de la ocs.

$\mathrm{El}$ actor fundamental en los vínculos entre ambas regiones ha sido el gobierno chino, que se ha mostrado como un elemento que aporta ideas y dinero. Fue el motor de la formación de la ocs y, con la firma de la Declaración sobre la Conducta de las Partes en el Mar del Sur de China en 2002 y su adhesión al Tratado de Amistad y Cooperación en el Sudeste de Asia en 2003, incrementó la predictibilidad en el escenario regional asiático. Esto permitió, al menos conceptualmente, unir al centro y sudeste de Asia en torno a China, como el factor esencial para la seguridad de esta macro región. De acuerdo con

18. Estos intereses, que en algunas ocasiones han coincidido con la agenda del combate internacional contra el terrorismo, no tienen su origen en septiembre de 2001. El problema que representan las redes de crimen organizado transnacional y las organizaciones fundamentalistas se planteó con antelación en la región, particularmente en Asia central con el ascenso de los talibanes en 1996, ante la aparente complacencia de las agencias gubernamentales estadounidenses. 
algunos analistas (Ermekbayev, 2011), esta unión, de llegar a ser realmente efectiva, tendría el potencial para convertirse en un "cinturón de seguridad" entre Europa y Asia. Bajo este escenario, China redimensionaría considerablemente su presencia internacional.

La relación entre ANSEA y la OCs ha despertado en Asia visiones contrapuestas. En un extremo, tenemos a quienes, como Timur Dadabaev (2007), sostienen que "construir esquemas institucionales similares a los de la Unión Europea o ANSEA en los estadios iniciales de la integración regional de Asia Central puede resultar improductivo, o peor, puede conducir a una derrota anunciada". En otro, existen quienes (Kosyrev, 2006), aseveran que el modelo de la ocs no es nuevo y que sus fundadores sencillamente tomaron el modelo de la ANSEA; sobre todo, enfatiza que en Asia central, al igual que en el sudeste, se ha puesto atención a los niveles de compatibilidad entre los socios, de tal manera que, tanto la estructura interna de la ocs como el sistema externo de sociedades son muy similares: adentro combinan ricos y otros no prósperos, mientras que hacia el exterior cuentan con una gran número de observadores. Hasta ahora ambas organizaciones, a diferentes niveles y de acuerdo a sus intereses, son muy activas hacia el exterior, lo mismo entre sí como respecto a Europa.

Ambos organismos, ANSEA y OCS, combinan flexibilidad en materia organizativa, con rigidez respecto a la membresía. Incluyen a un conjunto de países de diferentes dimensiones, sistemas políticos, grados de desarrollo y, pese a la vecindad, a veces de grandes diferencias culturales; pero sobre todo, de orientaciones ideológicas diametralmente opuestas en el pasado reciente y con perspectivas paralelas en relación a Estados Unidos, a quien no pueden hacer a un lado, pero tampoco desean tener demasiado cerca (al menos algunos de ellos). Los dos procesos, que se retroalimentan fortaleciéndose, conducen a cuestionar la importancia de la simetría como elemento central de la integración regional, la cual es alentada por un conjunto de elementos discursivos clave en un contexto poco ideologizado, pero no por ello menos competitivo.

La Ocs parece seguir de cerca los pasos de ANSEA, pero de convertirse en una copia se paralizaría, aunque su nivel de desarrollo ha pasado ya los momentos más difíciles. Un aspecto importante para la arquitectura regional es que la ACRSA, alentada por los avances organizativos en el centro y en el sudeste, así como por el crecimiento económico chino, ha emprendido "profundos cambios en su estructura organizacional y patrón operativo" (Fu, 2007). 
Este proceso inició en 2007 con el ingreso de Afganistán y la participación, por vez primera, de cinco observadores (China, Japón, Corea, Estados Unidos y la Unión Europea) durante su $14^{\circ}$ Cumbre. El interés de los miembros de la ACRSA por profundizar sus lazos económicos con China y, a la vez, con la región en general, fue una de las preocupaciones principales que los motivó a aceptar la participación de observadores. Con ello, la ACRSA se fortalece en el escenario político regional y China se reafirma como el eslabón clave en la cadena de organizaciones regionales asiáticas.

\section{Instituciones multifacéticas y redistribución de la relación de fuerzas: consideraciones finales}

Con base en el estudio de la dimensión institucional de la relación ANSEA-OCS realizado en este trabajo, es posible comprender que el regionalismo en Asia, en sus partes sudeste y central, ha evolucionado en cuatro décadas de algo cercano a un régimen internacional enfocado en la seguridad regional a un conjunto de instituciones regionales multifacéticas. Durante este proceso:

a) La construcción de instituciones regionales ha sido vista como el mecanismo idóneo para cristalizar los intereses específicos de cada Estado.

b) La antigua competencia "a secas" se transformó en competencia cooperativa gracias su reglamentación y a los avances en la disminución de la incertidumbre.

Podemos notar cómo los Estados no renuncian a establecerse como poderes. Por el contrario, enfatizan que su vigencia puede ser más importante dentro del contexto de una reconfiguración de los órdenes regionales que se caracteriza, entre otros aspectos, por la redistribución de la relación de fuerzas. Esta redistribución puede darse de una manera organizada fortaleciendo a las instituciones y a la cooperación entre ellas, con lo cual sería posible dimensionar sus posibilidades de liderazgo. En ese sentido, Beijing, más que ningún otro gobierno de la región, se perfila para tener un mejor lugar gracias a su papel motor en la ocs y su gran capacidad negociadora dentro de ANSEA.

\section{Bibliografía}

Acharya, Amitav (1999), "Realism, Institutionalism, and the Asian Economic Crisis”, Contemporary Southeast Asia, vol. 21, núm. 1, pp. 1-29. 
Aris, Stephen (2009), "A New Model of Asian Regionalism: does the Shanghai Cooperation Organization have more potential than ASEAN?", Cambridge Review of International Affairs, vol. 22, núm. 3, pp. 451-467.

ASEAN (2007), Chairperson's Statement of the 12th ASEAN Summit H.E. the President Gloria Macapagal Arroyo: "One caring and sharing community" (Cebu, Philippines, 13 January 2007). Disponible en: http://www.aseansec.org/19280.htm (consultado el 18 de octubre de 2012).

Beijing Review (1994), “China's Basic Policy towards Central Asia”, Beijing Review, vol. 37, núm. 18, pp. 18-19.

CCTV.COM English (2012), "Joint Sco military drill concludes with a success", (15 de junio de 2012). Disponible en: http://english.cntv. cn/20120615/106726.shtml. Fecha de consulta: octubre 20, 2012).

China.org.cn (2003), "China, Philippines Issue Joint Communique on Relations, World Issues”, ( $1^{\circ}$ de septiembre de 2003). Disponible en: http:// www.china.org.cn/english/international/73968.htm. Fecha de consulta: octubre 18, 2012).

Dadabaev, Timur (2007), "Central Asian Regional Integration: Between Reality and Myth", CACI Analyst, (2 de febrero de 2007). Disponible en: www.cacianalyst.org/newsite/?q=node/4604. Fecha de consulta: octubre 18, 2012.

Ermekbayev, N. B. (2011), "The Association of South East Asian Nations and the Shanghai Cooperation Organization: Opportunities and Perspectives of Interaction", The Journal of Turkish Weekly. Disponible en: http:// www.turkishweekly.net/article/269/the-association-of-south-east-asiannations-and-the-shanghai-cooperation-organization-opportunities-andperspectives-of-interaction.html. Fecha de consulta: octubre 18, 2012).

Fu, Xiaoqiang (2007), "South Asia Moving Toward Closer China Relations", China Daily, (3 de abril 2007). Disponible en: http://www.china.org.cn/ english/international/205899.htm. Fecha de consulta: octubre 18, 2012).

Haro, Francisco (2005), "Política exterior china en Asia Central: construcción del institucionalismo regional”, en Xulio Ríos (coord.) Política exterior de China. La diplomacia de una potencia emergente, Barcelona: Ediciones Bellaterra, pp. 191-217.

- - (2006), "La socialización internacional de la República Popular China: Una propuesta para su análisis”, en José Luis Estrada, José Luis León, Ricardo Buzo (eds.), China en el siglo Xxi. Economía, política y sociedad de una potencia emergente, México: UAM Iztapalapa y Miguel Ángel Porrúa, pp. 301 y 329. 
(2007), “Integración económica en el sureste de Asia: la formación del poder regional chino”, Aduanas (abril), pp. 38-41

He, Baogang (2003), "A Catalyst for Regional for Asian Regional Integration", ASEAN Focus Group (diciembre). Disponible en: http://www.chinesedemocratization.com/English\%20Website/frame/materials/journal/ english/A\%20Catalyst\%20for\%20Asian\%20Regional\%20Integration. htm. Fecha de consulta: diciembre 27, 2012).

Johnston, Alastair Iain (2003), "Socialization in International Institutions: The ASEAN Way and International Relations Theory", en G. John Ikenberry y Michael Mastanduno (eds.), International Relations Theory and the Asia-Pacific, Nueva York: Columbia University Press, pp. 107-162.

Kangas, Roger D. (1995), "State Building and Civil Society in Central Asia", en Vladimir Tismaneanu (coord.), Political Culture and Civil Society in Russia and the New States of Eurasia, Nueva York: M.E. Sharpe, p. 283. Keohane, Robert (1984), After Hegemony. Cooperation and Discord in the World Political Economy, Princeton: Princeton University Press.

- - (1986), "Reciprocity in International Relations", International Organization, vol. 40, núm. 1, pp. 1-27.

Kosyrev, Dimitri (2006), "Putin Has An Asian Vision For Growth And Cooperation”, United Press International, (20 de junio, 2006). Disponible en: www.spacewar.com/reports/Putin_Has_An_Asian_Vision_For_Growth_ And_Cooperation.html. Fecha de consulta: enero 2, 2012.

Narine, Shaun (2000), "Economics and Security in the Asia Pacific: A Constructivist Analysis", en Working Papers International Studies Association, 41st Annual Convention, Los Ángeles. Disponible en: http://www.ciaonet. org/isa/nas01/

People's Daily Online (2005), "Arroyo proposes ASEAN-SCO anti-terrorism cooperation", (13 de diciembre de 2005). Disponible en: http://english. peopledaily.com.cn/200512/13/eng20051213_227758.html. Fecha de consulta: octubre 18, 2012).

Perlez, Jane (2012), "Asian Leaders at Regional Meeting Fail to Resolve Disputes Over South China Sea", The New York Times, (12 de julio de 2012). Disponible en: http://www.nytimes.com/2012/07/13/world/asia/ asian-leaders-fail-to-resolve-disputes-on-south-china-sea-during-aseansummit.html?pagewanted=all. Fecha de consulta: diciembre 27, 2012).

Ramírez Bonilla, Juan José (2012), "Foro Regional ANSEA, ¿simiente de un nuevo orden global?”, en Francisco Javier Haro Navejas y Alfredo Román 
Zavala (coords.), Diez naciones en busca de liderazgo, México: Miguel Ángel Porrúa y UAM-I, pp. 33-70.

Shambaugh, David (2005), "The Evolving Asian System: Implications for the Regional Security Architecture", presentado en el $8^{\circ}$ Waldbroel Group Meeting on the European and Euro-Atlantic Coordination of Security Policies vis-à-vis the Asia Pacific, Berlín, 14-15 de diciembre de 2005. Disponible en: http://www.swp-berlin.org/fileadmin/contents/products/ projekt_papiere/Shambaugh2005_Evolving_AsianSystem_ks.pdf. Fecha de consulta: diciembre 27, 2012.

Tay, Simon (2009), "ASEAN remains important — Simon Tay", Singapore Institute of International Affairs, Commentaries (April 29, 2009). Disponible en: http://www.siiaonline.org/content.aspx?page=commentariesDetails \&id=176\&ArticleCategoryId=4. Fecha de consultado: diciembre 27, 2012. Wendt, Alexander (1994), "Collective Identity Formation and the International State”, American Political Science Review, vol. 88, núm. 2, pp. 384-396. 


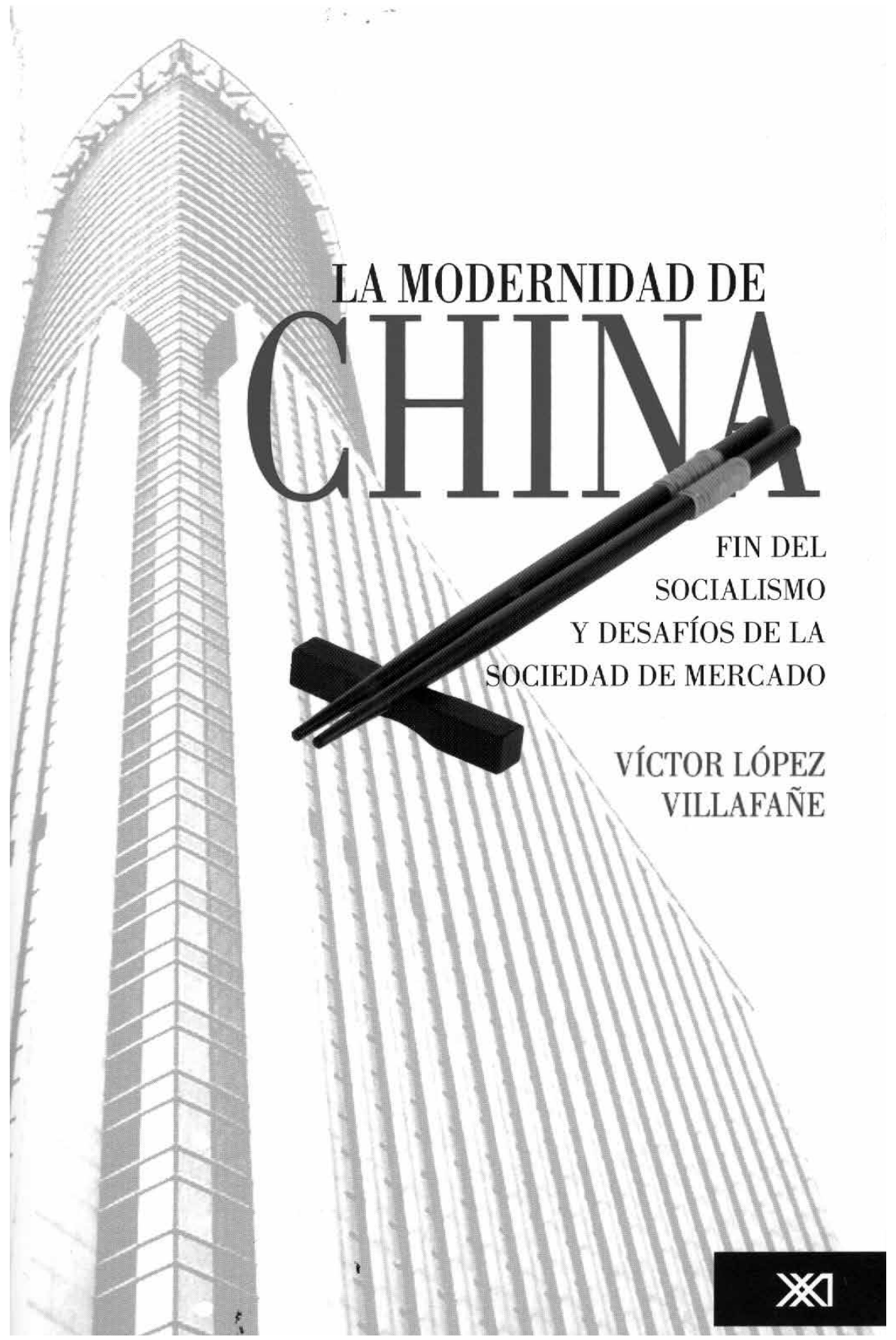

